\title{
Epistasis in Predator-Prey Relationships
}

\author{
Iuliia Inozemtseva, James Braselton* \\ Georgia Southern University, Statesboro, GA, USA \\ Email: ii00073@georgiasouthern.edu, “ibraselton@georgiasouthern.edu
}

Received 5 June 2014; revised 23 July 2014; accepted 8 August 2014

Copyright $@ 2014$ by authors and Scientific Research Publishing Inc.

This work is licensed under the Creative Commons Attribution International License (CC BY). http://creativecommons.org/licenses/by/4.0/

(c) ()

\begin{abstract}
Epistasis is the interaction between two or more genes to control a single phenotype. We model epistasis of the prey in a two-locus two-allele problem in a basic predator-prey relationship. The resulting model allows us to examine both population sizes as well as genotypic and phenotypic frequencies. In the context of several numerical examples, we show that if epistasis results in an undesirable or desirable phenotype in the prey by making the particular genotype more or less susceptible to the predator or dangerous to the predator, elimination of undesirable phenotypes and then genotypes occurs.
\end{abstract}

\section{Keywords}

Epistasis, Predator-Prey Equations, Logistic Equation, Hardy-Weinberg Equation

\section{Introduction}

The usual starting point for models in population genetics is a model of random mating for a one-locus, twoallele problem with Hardy-Weinberg proportions for the alleles. Refer to Karlin [1], for an overview of mathematical models of population genetics. In genetic problems, the genotype is defined to be the genetic makeup of an organism. For example, in a one-locus, two-allele model, one can label the alleles as $A$ (dominant) and $a$ (recessive). The possible genotypes are then $A A, A a$, and $a a$. On the other hand, the phenotype is defined to be the set of observable characteristics of an individual resulting from the interaction of its genotype with the environment. In the absence of epistasis, defined in the next paragraph, with the above genotypes, the phenotypes would be $A$ for the genotypes of type $A A$ and $A a$ and $a$ for the genotype of type $a a$.

Epistasis occurs when the genotype results in a phenotype different from that expected. For example, in the context of the example above, epistasis would occur if either the $A A$ or $A a$ genotype did not result in the phenotype expected, $A$.

One of the more interesting examples of epistasis in humans occurs in human blood in the form of the

${ }^{*}$ Corresponding author.

How to cite this paper: Inozemtseva, I. and Braselton, J. (2014) Epistasis in Predator-Prey Relationships. Open Journal of Applied Sciences, 4, 473-491. http://dx.doi.org/10.4236/ojapps.2014.49046 
Bombay Phenotype. The Bombay Phenotype is a very rare blood phenotype in humans that is scientifically referred to as the $h h$ (the $H$ ) antigen that results in a phenotype of type $O$, even though the genotype of these individuals is of type $A B O$, which typically would be expressed as of type $A B$. People with the Bombay Phenotype can donate to any member of the $A B O$ blood group system, but they can only receive blood from others with the Bombay Phenotype. The Bombay Phenotype is very rare and only occurs in approximately 1 in 10,000 individuals in India and 1 in a million people in Europe (see [2]).

In the study of epistasis discussed here the modifications introduced are that epistasis is incorporated into a standard predator-prey model, so that one genotype of the prey may be more or less susceptible to the predator than other prey with a different genotype, but the same phenotype in the absence of epistasis. Second, we introduce epistasis in the standard predator-prey model so that one genotype of the prey is dangerous (lethal or poisonous) to the predator than other prey with different genotypes but the same expected phenotype in the absence of epistasis.

This is accomplished by a perturbation of the random mating model within the context of established ecological models for the evolution of a predator-prey population.

Of course, general mating preferences almost certainly depend on numerous external factors that might include but are not limited to available mates, parasites, season, climate, and such that they are beyond the scope of the model discussed here, but could lead to interesting extensions of the situations discussed here.

\section{Formulation of the Model}

\subsection{The Standard Predator-Prey Equations}

Let $x=x(t)$ denote the size or density of the prey population and $y=y(t)$ denote the size or density of the predator population. Then the standard predator-prey equations take the form

$$
\begin{aligned}
& x^{\prime}=x(a-k y)=x a-k x y \\
& y^{\prime}=y(-c+d x)=d x y-c y \\
& x(0)=x_{0}, \quad y(0)=y_{0},
\end{aligned}
$$

where $a, k, c$, and $d$ are all positive constants. Refer to Lotka [3], or Karlin [1], for basic details regarding the standard Predator-Prey model. In the absence of the predator, the prey has linear growth, given by the $x^{\prime}=a x$ term. The death rate of the prey is governed by predator-prey interactions given by the quadratic $-k x y$ term, which contributes to the growth rate of the predator by the $d x y$ term. We interpret $k$ to be the catchability of the prey $x$ by the predator $y$. Details regarding the standard predator-prey equations and different interpretations of the coefficients are discussed in most introductory differential equations texts like Abell and Braselton [4], or introductory mathematical modeling and/or mathematical biology texts such as Beltrami [5], or Murray [6].

The most important result for system (1) is that the equilibrium (rest) point $\left(x_{0}, y_{0}\right)=(c / d, a / k)$ is classified as a center in the corresponding linearized system because the eigenvalues of the Jacobian of system (1),

$$
\boldsymbol{J}(x, y)=\left(\begin{array}{ll}
\frac{\partial}{\partial x}(x a-k x y) & \frac{\partial}{\partial y}(x a-k x y) \\
\frac{\partial}{\partial x}(d x y-c y) & \frac{\partial}{\partial y}(d x y-c y)
\end{array}\right)=\left(\begin{array}{cc}
a-k y & -k x \\
d y & -c+d x
\end{array}\right)
$$

evaluated at the equilibrium (rest) point $\left(x_{0}, y_{0}\right)=(c / d, a / k)$ are

$$
\lambda_{1,2}=\left|\begin{array}{cc}
a-k y & -k x \\
d y & -c+d x
\end{array}\right|_{\left(x_{0}, y_{0}\right)=(c / d, a / k)}=\left|\begin{array}{cc}
0 & -c k / d \\
a d / k & 0
\end{array}\right|= \pm i \sqrt{a c} .
$$

While the stability of the equilibrium point in the nonlinear system is generally inconclusive in this case, other solution methods can verify that the equilibrium point is, in fact, a center. A typical example is shown in Figure 1, where we have used the values $a=2, k=-1, c=-3$, and $d=1$. In Figure 1, observe how the limit cycles revolve about the center, $\left(x_{0}, y_{0}\right)=(3,2)$. 


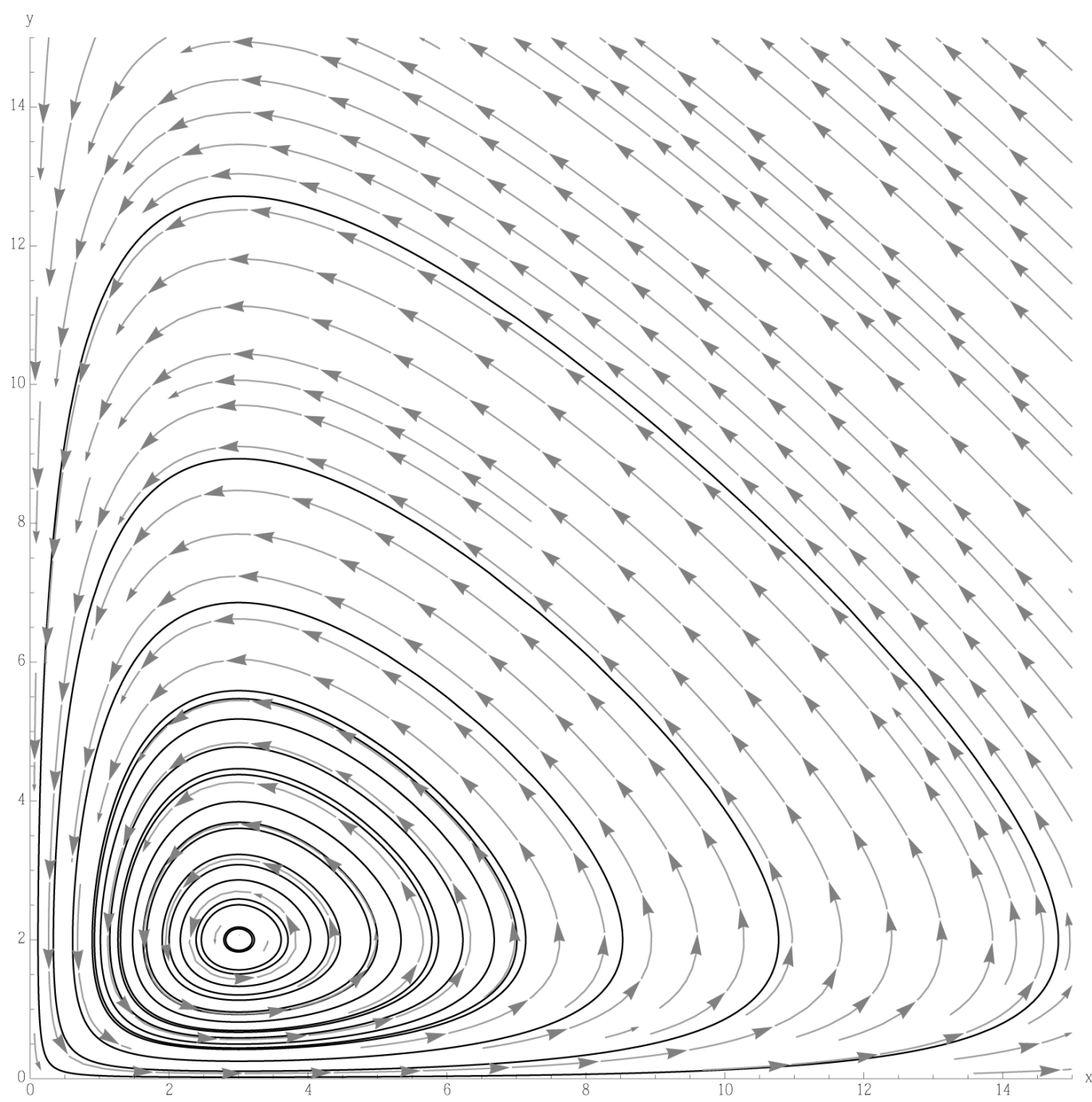

Figure 1. We choose $a=2, k=-1, c=-3$, and $d=1$. Observe how the solution curves revolve about the center, $\left(x_{0}, y_{0}\right)=(3,2)$.

\subsection{A Two-Locus, Two-Allele Model}

In the two-locus, two-allele problem, the number of genotypes is nine but in the absence of epistasis, the number of phenotypes is four. If the $A$ and $B$ alleles are dominant, the expected result is four phenotypes $A B\left(x_{1}\right.$, $x_{2}, x_{4}$, and $\left.x_{5}\right), A b\left(x_{3}\right.$ and $\left.x_{6}\right), a B\left(x_{7}\right.$ and $\left.x_{8}\right)$, and $a b\left(x_{9}\right)$, as described next. For the two-locus, two-allele problem, we consider a population $x=x(t)$ with size (or density)

$$
x=x_{1}+x_{2}+x_{2}+x_{3}+x_{4}+x_{5}+x_{6}+x_{7}+x_{8}+x_{9}
$$

where

- $x_{1}$ is the size of the population of type $A A B B$ (expected phenotype $A B$ ),

- $x_{2}$ is the size of the population of type $A A B b$ (expected phenotype $A B$ ),

- $x_{3}$ is the size of the population of type $A A b b$ (expected phenotype $A b$ ),

- $x_{4}$ is the size of the population of type $A a B B$ (expected phenotype $A B$ ),

- $x_{5}$ is the size of the population of type $A a B b$ (expected phenotype $A B$ ),

- $x_{6}$ is the size of the population of type $A a b b$ (expected phenotype $A b$ ),

- $x_{7}$ is the size of the population of type $a a B B$ (expected phenotype $a B$ ),

- $x_{8}$ is the size of the population of type $a a B b$ (expected phenotype $a B$ ) and 
- $x_{9}$ is the size of the population of type $a a b b$ (expected phenotype $a b$ ).

The proportion of gametes of type $A B, A b, a B$, and $a b$ are given by

$$
\begin{aligned}
& p_{x}=\frac{1}{x}\left(x_{1}+\frac{1}{2}\left(x_{2}+x_{4}\right)+\frac{1}{4} x_{5}\right), \\
& q_{x}=\frac{1}{x}\left(x_{3}+\frac{1}{2}\left(x_{2}+x_{6}\right)+\frac{1}{4} x_{5}\right), \\
& r_{x}=\frac{1}{x}\left(x_{7}+\frac{1}{2}\left(x_{4}+x_{8}\right)+\frac{1}{4} x_{5}\right),
\end{aligned}
$$

and

$$
s_{x}=\frac{1}{x}\left(x_{9}+\frac{1}{2}\left(x_{6}+x_{8}\right)+\frac{1}{4} x_{5}\right),
$$

respectively. Observe that $p_{x}+q_{x}+r_{x}+s_{x}=1$. Table A1 in the Appendix shows the expected ratio of offspring produced by each $x_{i}-x_{j}$ combination. Refer to articles like Braselton et al. [7], or Szathmáry [8], for details regarding these and similar calculations.

Assuming random mating between the genotypes of the prey, the predator-prey Equations (1) become

$$
\begin{aligned}
& x_{1}^{\prime}=\operatorname{axp}^{2}-k_{1} x_{1} y \\
& x_{2}^{\prime}=2 a x p q-k_{2} x_{2} y \\
& x_{3}^{\prime}=a x q^{2}-m_{3} x_{3} y c \\
& x_{4}^{\prime}=2 a x p r-k_{4} x_{4} y \\
& x_{5}^{\prime}=2 a x(p s+r q)-k_{5} x_{5} y \\
& x_{6}^{\prime}=2 a x q s-m_{6} x_{6} y \\
& x_{7}^{\prime}=a x r^{2}-f_{7} x_{7} y \\
& x_{8}^{\prime}=2 a x r s-f_{8} x_{8} y \\
& x_{9}^{\prime}=a x s^{2}-l_{9} x_{9} y \\
& y^{\prime}=y(-c+d x)
\end{aligned}
$$

with initial conditions

$$
\begin{aligned}
& x_{1}(0)=x_{10}, x_{2}(0)=x_{20}, x_{3}(0)=x_{30}, x_{4}(0)=x_{40}, x_{5}(0)=x_{50}, \\
& x_{6}(0)=x_{60}, x_{7}(0)=x_{70}, x_{8}(0)=x_{80}, x_{9}(0)=x_{90}, y(0)=y_{0},
\end{aligned}
$$

where we have used Table A1 in the Appendix to compute the coefficients and simplified the results using Equation (2) as well as omitted the subscripts for the $p=p_{x}, q=q_{x}, r=r_{x}$, and $s=s_{x}$ terms.

If $k=k_{1,2,4,5}=m_{3,6}=f_{7,8}=l_{9}$, adding system (3) and then substituting the proportion of gametes given in Equation (2) and adding $x=x_{1}+x_{2}+x_{2}+x_{4}+x_{5}+x_{6}+x_{7}+x_{8}+x_{9}$ results in the predator-prey equations, system (1). Using Equation (2), the allele frequencies of $A, a, B$, and $b$ are given by

$$
\begin{aligned}
& \frac{1}{x}\left(x_{1}+x_{2}+x_{3}+\frac{1}{2}\left(x_{4}+x_{5}+x_{6}\right)\right)=p+q, \\
& \frac{1}{x}\left(x_{7}+x_{8}+x_{9}+\frac{1}{2}\left(x_{4}+x_{5}+x_{6}\right)\right)=r+s, \\
& \frac{1}{x}\left(x_{1}+x_{4}+x_{7}+\frac{1}{2}\left(x_{2}+x_{5}+x_{8}\right)\right)=p+r, \\
& \text { and } \\
& \frac{1}{x}\left(x_{3}+x_{6}+x_{9}+\frac{1}{2}\left(x_{2}+x_{5}+x_{8}\right)\right)=q+s,
\end{aligned}
$$


respectively.

In addition, using system (3) and Equations (2), we have

$$
\begin{aligned}
& \frac{x_{1}^{\prime}+x_{2}^{\prime}+x_{3}^{\prime}+\frac{1}{2}\left(x_{4}^{\prime}+x_{5}^{\prime}+x_{6}^{\prime}\right)}{x_{1}+x_{2}+x_{3}+\frac{1}{2}\left(x_{4}+x_{5}+x_{6}\right)}-\frac{x^{\prime}}{x}=0, \\
& \frac{x_{7}^{\prime}+x_{8}^{\prime}+x_{9}^{\prime}+\frac{1}{2}\left(x_{4}^{\prime}+x_{5}^{\prime}+x_{6}^{\prime}\right)}{x_{7}+x_{8}+x_{9}+\frac{1}{2}\left(x_{4}+x_{5}+x_{6}\right)}-\frac{x^{\prime}}{x}=0, \\
& \frac{x_{1}^{\prime}+x_{4}^{\prime}+x_{7}^{\prime}+\frac{1}{2}\left(x_{2}^{\prime}+x_{5}^{\prime}+x_{8}^{\prime}\right)}{x_{1}+x_{4}+x_{7}+\frac{1}{2}\left(x_{2}+x_{5}+x_{8}\right)}-\frac{x^{\prime}}{x}=0, \\
& \frac{x_{3}^{\prime}+x_{6}^{\prime}+x_{9}^{\prime}+\frac{1}{2}\left(x_{2}^{\prime}+x_{5}^{\prime}+x_{8}^{\prime}\right)}{x_{3}+x_{6}+x_{9}+\frac{1}{2}\left(x_{2}+x_{5}+x_{8}\right)}-\frac{x^{\prime}}{x}=0 .
\end{aligned}
$$

Integrating and exponentiating each Equation in (6) results in the following

$$
p+q=p(0)+q(0), p+r=p(0)+r(0), q+s=q(0)+s(0), \text { and } r+s=r(0)+s(0) .
$$

This proves the theorem.

Theorem 1. For random mating, the relative frequencies of the alleles $A, a, B$, and $b$ are constant, agreeing with the Hardy-Weinberg equation.

Theorem 2. If $k=k_{1,2,4,5}=m_{3,6}=f_{7,8}=l_{9}$, there are up to 14 equilibrium (rest) points, provided that the appropriate quantities are nonnegative.

Proof. In the following, $x_{1}=x_{1}(0), x_{2}=x_{2}(0), x_{3}=x_{3}(0), x_{4}=x_{4}(0), x_{5}=x_{5}(0), x_{6}=x_{6}(0)$, $x_{7}=x_{7}(0), x_{8}=x_{8}(0)$, and $x_{9}=x_{9}(0)$.

$E_{1,2}$ are given by

$$
\begin{gathered}
x_{1}^{*}= \pm \frac{x_{5}\left(2 \sqrt{2} \sqrt{c x_{5}^{2} x_{8}\left(2 c x_{8}-d\left(x_{5}+2 x_{8}\right)^{2}\right)} \pm 4 c x_{5} x_{8}+d x_{5}\left(x_{5}+2 x_{8}\right)^{2}\right)}{8 d x_{8}\left(x_{5}+2 x_{8}\right)^{2}} \\
x_{2}^{*}=\frac{x_{5}^{2}}{4 x_{8}}, \\
\left.x_{3}^{*}=-\frac{d x_{5}^{3}\left(x_{5}+2 x_{8}\right)^{2}}{8 x_{8}\left(\mp 2 \sqrt{2} \sqrt{c x_{5}^{2} x_{8}\left(2 c x_{8}-d\left(x_{5}+2 x_{8}\right)^{2}\right)}-4 c x_{5} x_{8}+d x_{5}\left(x_{5}+2 x_{8}\right)^{2}\right.}\right) \\
x_{4}^{*}= \pm \frac{2 \sqrt{2} \sqrt{c x_{5}^{2} x_{8}\left(2 c x_{8}-d\left(x_{5}+2 x_{8}\right)^{2}\right)} \pm 4 c x_{5} x_{8}+d x_{5}\left(x_{5}+2 x_{8}\right)^{2}}{2 d\left(x_{5}+2 x_{8}\right)^{2}}, \\
x_{5}^{*}=x_{5}, \\
x_{6}^{*}= \pm \frac{d x_{5}^{2}\left(x_{5}+2 x_{8}\right)^{2}}{4 \sqrt{2} \sqrt{c x_{5}^{2} x_{8}\left(2 c x_{8}-d\left(x_{5}+2 x_{8}\right)^{2}\right)} \pm 8 c x_{5} x_{8} \mp 2 d x_{5}\left(x_{5}+2 x_{8}\right)^{2}},
\end{gathered}
$$




$$
\begin{gathered}
x_{7}^{*}=-\frac{x_{8}\left(\mp 2 \sqrt{2} \sqrt{c x_{5}^{2} x_{8}\left(2 c x_{8}-d\left(x_{5}+2 x_{8}\right)^{2}\right)}-4 c x_{5} x_{8}+d x_{5}\left(x_{5}+2 x_{8}\right)^{2}\right)}{2 d x_{5}\left(x_{5}+2 x_{8}\right)^{2}}, \\
x_{8}^{*}=x_{8}, \\
x_{9}^{*}= \pm \frac{d x_{5} x_{8}\left(x_{5}+2 x_{8}\right)^{2}}{4 \sqrt{2} \sqrt{c x_{5}^{2} x_{8}\left(2 c x_{8}-d\left(x_{5}+2 x_{8}\right)^{2}\right)} \pm 8 c x_{5} x_{8} \mp 2 d x_{5}\left(x_{5}+2 x_{8}\right)^{2}},
\end{gathered}
$$

and

$$
y^{*}=\frac{a}{k}
$$

Next, $E_{3,4}$ are given by

$$
\begin{gathered}
x_{1}^{*}=0, \quad x_{2}^{*}=0, \quad x_{3}^{*}=0, \quad x_{4}^{*}=0, \quad x_{5}^{*}=0, \quad x_{6}^{*}=0, \\
x_{7}^{*}=\frac{c-d x_{8} \pm \sqrt{c\left(c-2 d x_{8}\right)}}{2 d}, \quad x_{8}^{*}=x_{8}, \quad x_{9}^{*}=\frac{c-d x_{8} \mp \sqrt{c\left(c-2 d x_{8}\right)}}{2 d}, \quad \text { and } \quad y^{*}=\frac{a}{k} .
\end{gathered}
$$

$E_{5,6}$ are given by

$$
\begin{aligned}
& x_{1}^{*}=0, \quad x_{2}^{*}=0, \quad x_{4}^{*}=0, \quad x_{5}^{*}=0, \quad x_{6}^{*}=x_{6}, \quad x_{7}^{*}=0, \quad x_{8}^{*}=0, \\
& y^{*}=\frac{a}{k}, \quad x_{3}^{*}=\frac{c-d x_{6} \mp \sqrt{c\left(c-2 d x_{6}\right)}}{2 d}, \quad \text { and } \quad x_{9}^{*}=\frac{c-d x_{6} \mp \sqrt{c\left(c-2 d x_{6}\right)}}{2 d} .
\end{aligned}
$$

$E_{7,8}$ are given by

$$
\begin{aligned}
& x_{2}^{*}=0, \quad x_{3}^{*}=0, \quad x_{4}^{*}=x_{4}, \quad x_{5}^{*}=0, \quad x_{6}^{*}=0, \quad x_{8}^{*}=0, \quad x_{9}^{*}=0, \\
& y^{*}=\frac{a}{k}, \quad x_{1}^{*}=\frac{c-d x_{4} \mp \sqrt{c\left(c-2 d x_{4}\right)}}{2 d}, \quad \text { and } \quad x_{7}^{*}=\frac{c-d x_{4} \mp \sqrt{c\left(c-2 d x_{4}\right)}}{2 d} .
\end{aligned}
$$

$E_{9,10}$ are given by

$$
\begin{aligned}
& x_{2}^{*}=x_{2}, \quad x_{4}^{*}=0, \quad x_{5}^{*}=0, \quad x_{6}^{*}=x_{6}, \quad x_{7}^{*}=0, \quad x_{8}^{*}=0, \quad x_{9}^{*}=0, \\
& y^{*}=\frac{a}{k}, \quad x_{1}^{*}=\frac{c-d x_{2} \mp \sqrt{c\left(c-2 d x_{2}\right)}}{2 d}, \quad \text { and } \quad x_{3}^{*}=\frac{c-d x_{2} \mp \sqrt{c\left(c-2 d x_{2}\right)}}{2 d} .
\end{aligned}
$$

$E_{11}$ is given by $x_{1}^{*}=0, x_{2}^{*}=0, x_{3}^{*}=0, x_{4}^{*}=0, x_{5}^{*}=0, x_{6}^{*}=0, x_{7}^{*}=0, x_{8}^{*}=0, x_{9}^{*}=c / d$, and $y^{*}=a / k$.

$E_{12}$ is given by $x_{1}^{*}=0, x_{2}^{*}=0, x_{3}^{*}=c / d, x_{4}^{*}=0, x_{5}^{*}=0, x_{6}^{*}=0, x_{7}^{*}=0, x_{8}^{*}=0, x_{9}^{*}=0$, and $y^{*}=a / k$.

$E_{13}$ is given by $x_{1}^{*}=0, x_{2}^{*}=0, x_{3}^{*}=0, x_{4}^{*}=0, x_{5}^{*}=0, x_{6}^{*}=0, x_{7}^{*}=c / d, x_{8}^{*}=0, x_{9}^{*}=0$, and $y^{*}=a / k$.

Finally, $E_{14}$ is given by $x_{1}^{*}=c / d, x_{2}^{*}=0, x_{3}^{*}=0, x_{4}^{*}=0, x_{5}^{*}=0, x_{6}^{*}=0, x_{7}^{*}=0, x_{8}^{*}=0, x_{9}^{*}=0$, and $y^{*}=a / k$.

For all 14 rest points, $x_{1}^{*}+x_{2}^{*}+x_{3}^{*}+x_{4}^{*}+x_{5}^{*}+x_{6}^{*}+x_{7}^{*}+x_{8}^{*}+x_{9}^{*}=c / 9$.

Observe that the Jacobian, $\boldsymbol{J}$, for system (3) is a $10 \times 10$ matrix. We are unable to compute the eigenvalues of $\boldsymbol{J}$ at $E_{1}$ or $E_{2}$. However, for the remaining rest points (equilibrium points), $E_{4}, E_{5}, \cdots, E_{12}$, the eigenvalues of $\boldsymbol{J}$ evaluated at $E_{i}$ are $\lambda_{1,2}=0, \lambda_{3,4,5,6,7}=-a, \lambda_{8}=-a / 2$, and $\lambda_{9,10}= \pm i \sqrt{a c}$. Thus, we expect the rest points to usually be "center-like", which is illustrated in the computations.

When $k_{1}=k_{2}=k_{4}=k_{5}=k$ (phenotype $A B$ ), $m_{3}=m_{6}=m$ (phenotype $A b$ ), or $f_{7}=f_{8}=f$ (phenotype $a B$ ), the interpretation is that the corresponding phenotype of the prey have the same catchability to the predator. In this situation, we are not able to find exact formulas for the rest points as in the case when 
$k=k_{1,2,4,5}=m_{3,6}=f_{7,8}=l_{9}$. Thus, we conduct numerous numerical studies to explore some of the possibilities. When all parameter values have similar values as in the standard predator-prey Equation (1), we typically see a limit cycle that is illustrated in Figure 2.

Reviewing the standard predator-prey system of Equation (1), we expect to see that the higher parameter values of $a$ and $c$ (such as $a=2$ and $c=3$ ) give the advantage to the prey because the parameter $a$ is the growth rate of the (prey) species $x$ and the parameter $c$ is the death (or emigration) rate of (predator) species $y$.

With these parameter values, Figure 3 illustrates that the genotype $A a B b$ generally has the highest population sizes/densities. Because $A a B b-A a B b$ matings produce all of the other genotypes, all genotypes and phenotypes, $A B, A b, a B$ and $a b$ coexist.

Choosing values for $k$ and $m$ to be greater than values for $f$ and $l$ (using the same initial conditions and values for $a, b$, and $c$ in (1)) causes the two-locus, two-allele problem to typically result in a stable solution, such as the stable equilibrium point illustrated in Figure 4 and Figure 5.

Giving the "weakest" genotype, usually the genotype of type $a a b b$ (expected phenotype is $a b$ ), an advantage with a low catchability rate with respect to the other genotypes, such as $l=0.8$, helps this genotype to persist, even with relatively low population size/density. Regardless, in this simulation the genotype of type $A a B b$ again has the highest population density as illustrated in Figure 6.

From Figure 6 we see the stabilization points (or equilibrium or rest points) for the predator and prey populations. In Figure 7, the population density for the prey (black) converges to $x=3$ and the population
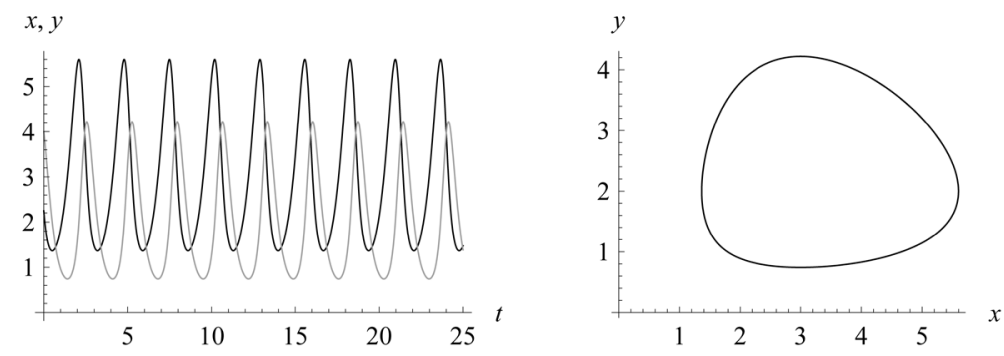

Figure 2. We choose $b=k=m=f=l=1$ and $a=2, c=3$. Initial values are $x_{10}=x_{20}=x_{30}=x_{40}=x_{50}=x_{60}=x_{70}=x_{80}=x_{90}=0.25, y_{0}=4$. All solutions, except for the equilibrium solution $x=3, y=2$, are periodic so all solution curves are closed curves in the graph on the right.

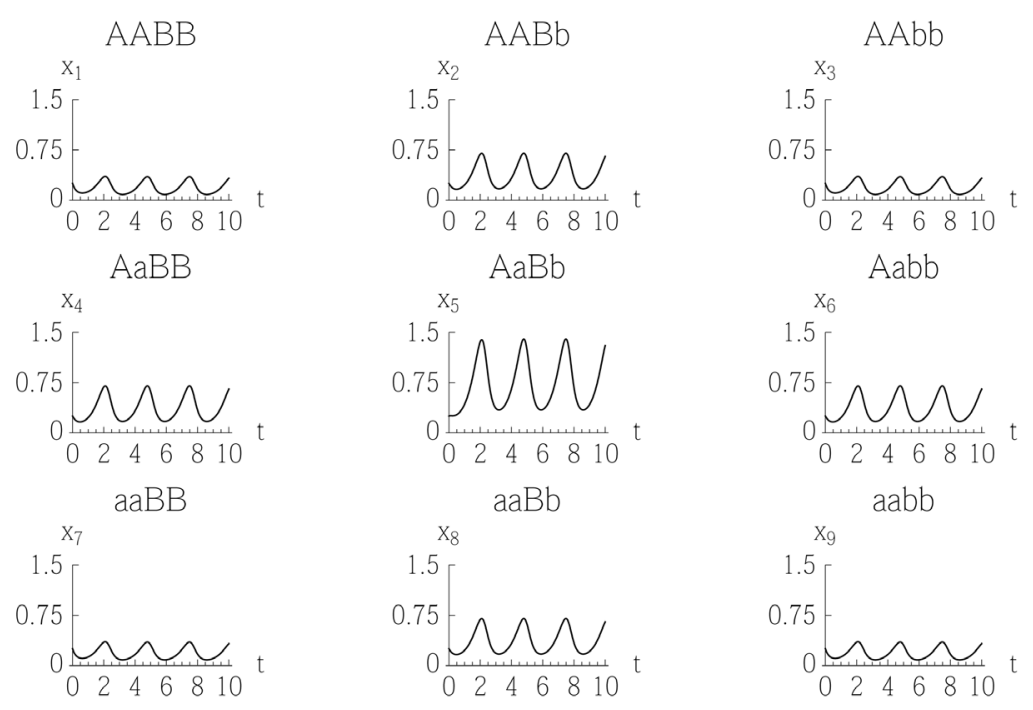

Figure 3. Because the genotype of type $A a B b$ has an advantage over the others, all genotypes coexist because random matings of this genotype produce all other genotypes (refer to the Appendix). 

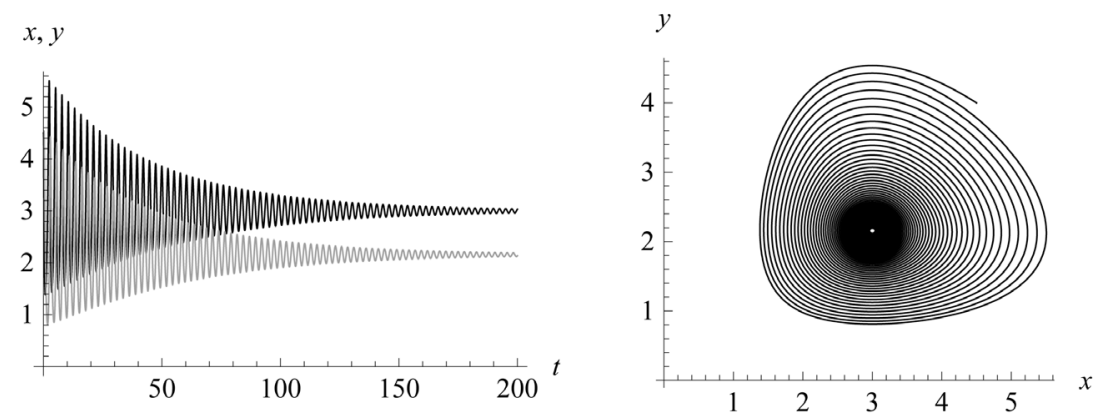

Figure 4. The parameter values used are $k=1, m=2, f=0.8$ and $l=0.8$, which result in stabilization.
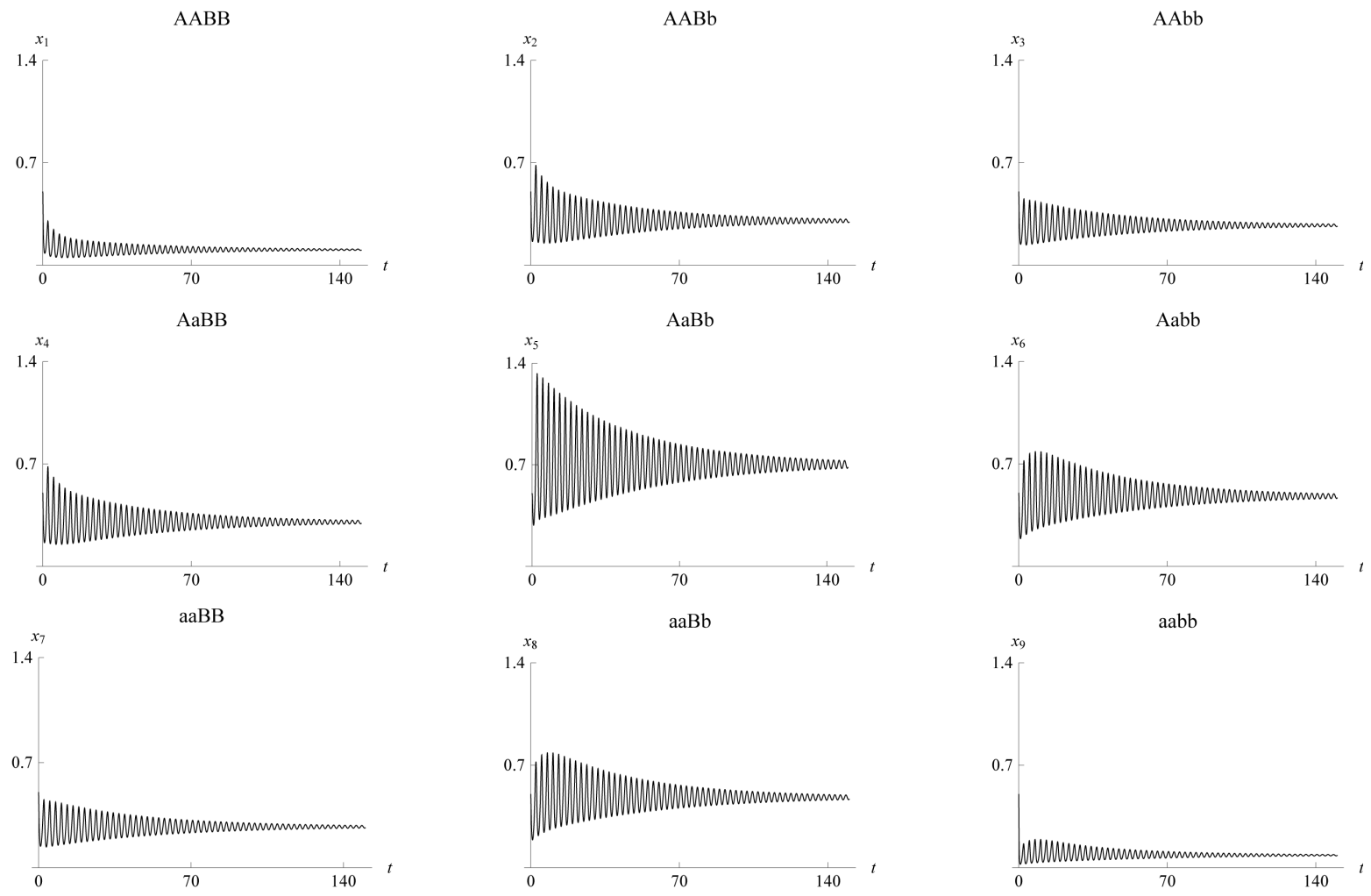

Figure 5. Stabilization with $k=1, m=2, f=0.8$ and $l=0.8$. The size/density of the population of each expected phenotype stabilizes.
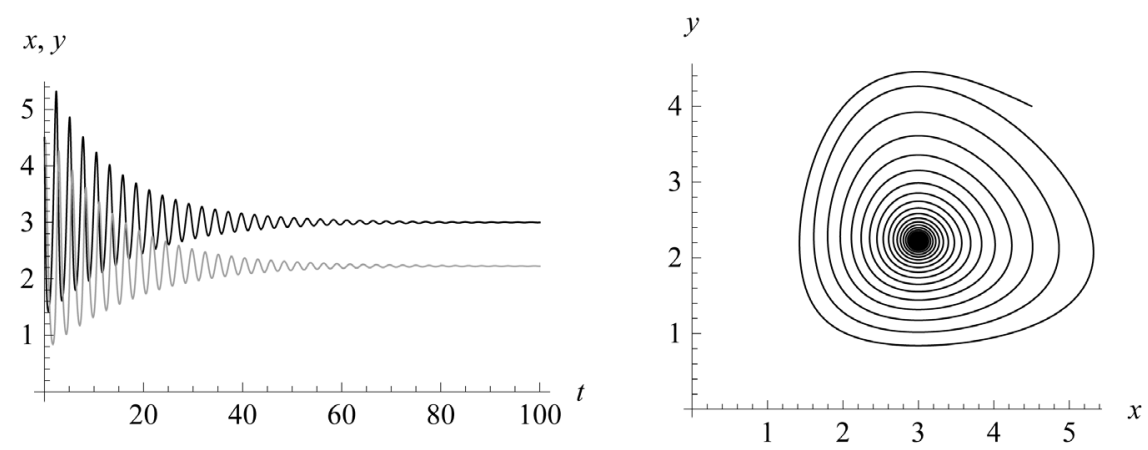

Figure 6. Stabilization with $k=1.2, \quad m=3, f=0.7$ and $l=0.7$. 

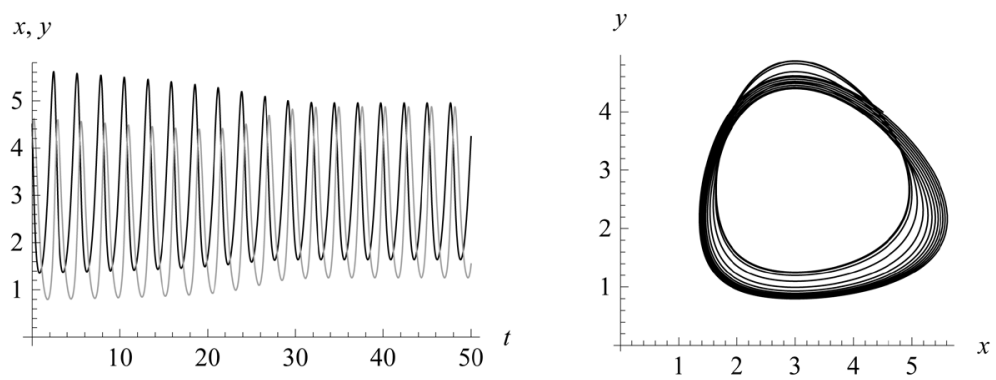

Figure 7. A limit cycle with $k=0.9, \quad m=f=1$ and $l=0.75$.

density of the predator (grey) converges to $y=2.1$.

Our final example using this model illustrates that when the expected weakest genotype of type, $a a b b$, dominates, both the $a$ and $b$ alleles may go to fixation (In the gene pool, fixation means that of two variants of a particular allele (gene), only one of the alleles remains after a period of time). Refer to Figure 7. Both prey and predator populations/densities remain at almost oscillatory rates as shown in the graph on the left in Figure 7.

In Figure 8, observe that we expected the "strongest" (lowest mortality rate than the other prey genotypes experience with the predator) genotypes to survive, but their population rates were continuously decreasing. At some point, around $x_{i}=30$, all vanish. At the same time, the population density of the $a a b b$ genotype type starts to increase and stabilizes in the range from 1.8 to 5: the $a$ and $b$ alleles go to fixation.

\section{Epistasis}

As discussed previously, epistasis occurs when the genotype results in a phenotype different from that expected. We model epistasis in a predator-prey relationship by forcing the catchability (given by the $k_{i}, m_{i}$, or $f_{i}$ terms) of one genotype of a particular phenotype to be greater or smaller than other organisms with the same expected phenotype. Recall that the $x_{i}$ (or $x_{i}^{\prime}$ ) (prey) and $y$ (predator) population densities are given by Equations (3) and that $a$ is the growth rate of species $x_{i}$ (prey) while $c$ is the death (or emigration) rate of species $y$ (predator) (Figure 9).

We use the same initial conditions and parameter values for $a, b$, and $c$ as in the previous simulations.

\subsection{Example 1. $k_{5}$ and $l_{9}$ Are the Greatest}

Setting $k_{5}$ greater than the other $k_{i}$ values models epistasis by giving the genotype $A a B b$ (expected phenotype $A B$ ) a higher prey-induced death rate than the other organisms with phenotype $A B$. We choose $l_{9}$ to be large as well because some would argue that the $a b$ phenotype would often be the weakest, which we continue to assume throughout the examples unless otherwise stated. Figure 10 illustrates that the population rate of the type $A A B B$ genotype (expected phenotype $A B)$ stabilizes around the point $\left(x_{1}=0.006\right)$. This happens because of several factors: the catchability of this genotype is high $\left(k_{1}=1.2\right)$ and population rates for the genotypes of type $A A B b, A a B B$ and $A a B b$ (same expected phenotype $A B$ ) are low. The population size rate for the genotype of type $a a b b$ with high catchability parameter $l_{9}=3$ stabilizes at the point around $x_{5}=0.15$. The organisms with genotypes $A a b b$ and $a a B B$ have the highest population rates (around 0.9 ).

\subsection{Example 2. (a) $k_{1}$ and $l_{9}$ Are the Greatest}

Setting $k_{1}$ greater than the other $k_{i}$ values models epistasis by giving the genotype $A A B B$ (expected phenotype $A B$ ) a higher prey-induced death rate than the other organisms with phenotype $A B$. Using these parameters, stabilization took nearly twice as long as in the previous model. Refer to Figure 11 and Figure 12.

The high value of the catchability parameter $k_{1}=3$ forces the organism of genotype $A A B B$ to very small levels, faster than in the previous example. The population size rate for the organisms of type $a a b b$ with catchability parameter $l_{9}=3$ is smaller too (around 0.1 ). On the other hand, the organisms with lower catchability values (genotypes $A A b b, A a b b, a a B B$, and $a a B b$ ) have the highest population densities.

Since $k_{5}=1.2$ and mating between the organism of type $A a B b$ produces all the other genotypes, Aabb 


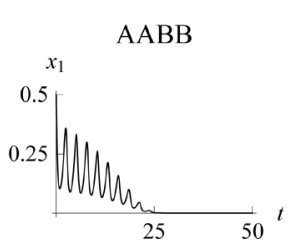

AaBB

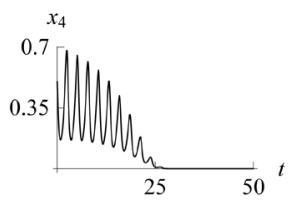

aaBB

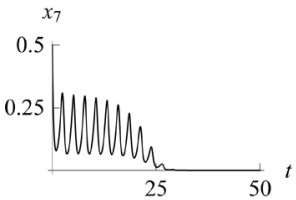

$\mathrm{AABb}$

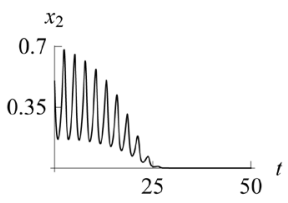

$\mathrm{AaBb}$

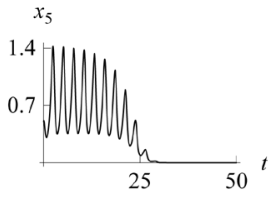

$\mathrm{aaBb}$

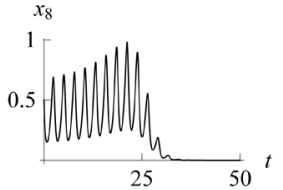

AAbb

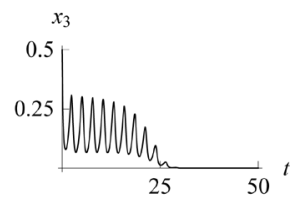

Aabb
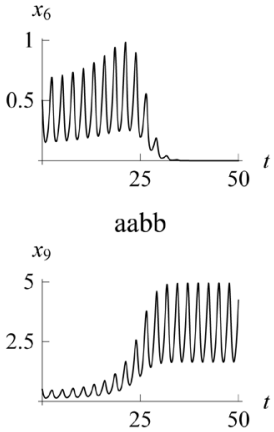

Figure 8. Limit cycles with $k=0.9, m=f=1$ and $l=0.75$. The organism of genotype $a a b b$ survives; the $a$ and $b$ alleles go to fixation.
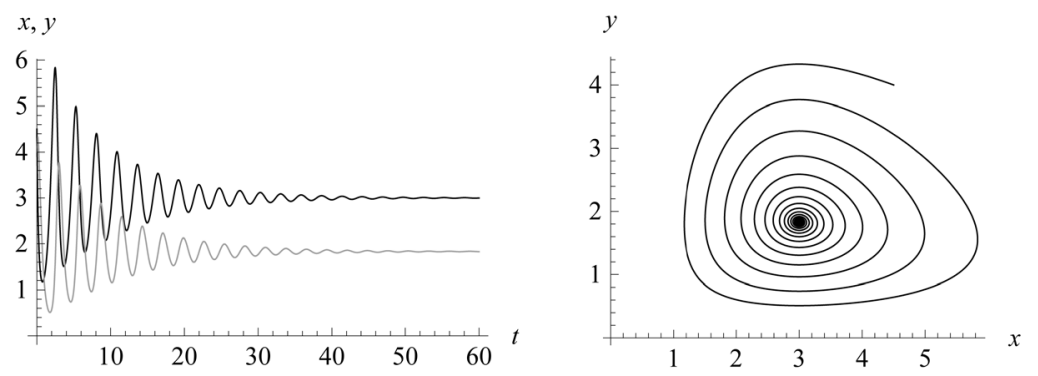

Figure 9. Stabilization with $k_{1}=1.2, k_{2}=1.2, k_{4}=1.2, k_{5}=3, l_{9}=3, m_{3}=0.8$, $m_{6}=0.8, f_{7}=0.8, f_{8}=0.8$.
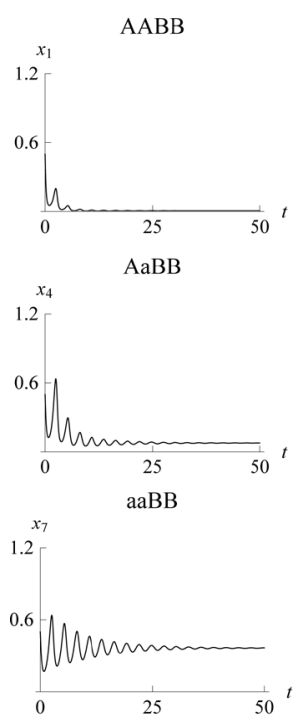
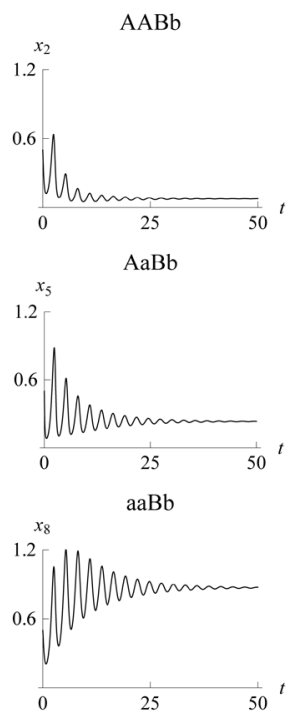

AAbb

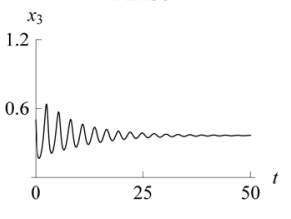

Aabb

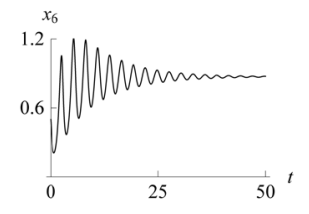

$\mathrm{aabb}$

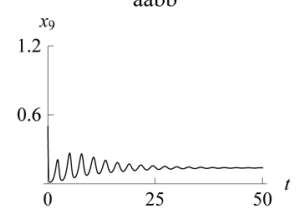

Figure 10. Stabilization with $k_{1}=1.2, k_{2}=1.2, k_{4}=1.2, k_{5}=3, l_{9}=3, m_{3}=0.8$, $m_{6}=0.8, f_{7}=0.8, f_{8}=0.8$. 

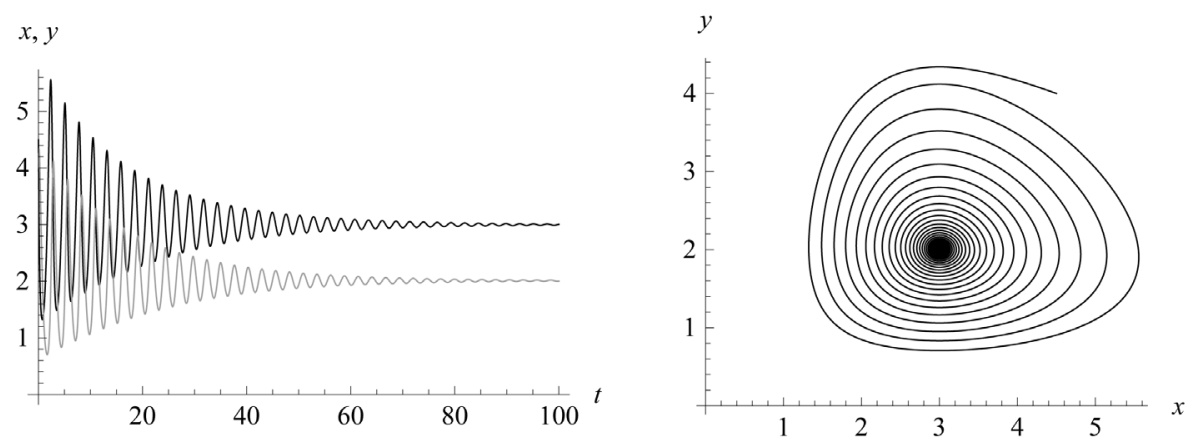

Figure 11. Stabilization with $k_{1}=3, k_{2}=1.2, k_{4}=1.2, k_{5}=1.2, l_{9}=3, m_{3}=0.8, m_{6}=0.8$, $f_{7}=0.8, f_{8}=0.8$.

AABB

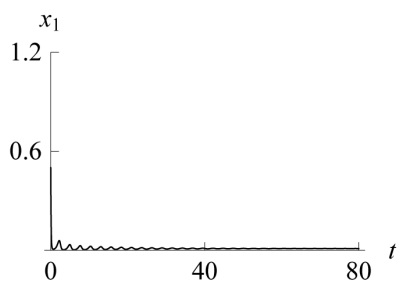

$\mathrm{AaBB}$
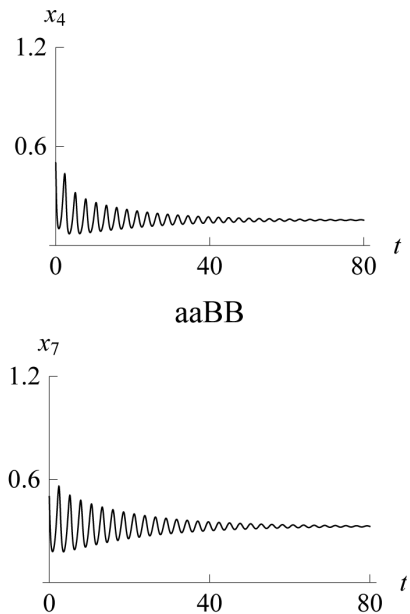

$\mathrm{AABb}$

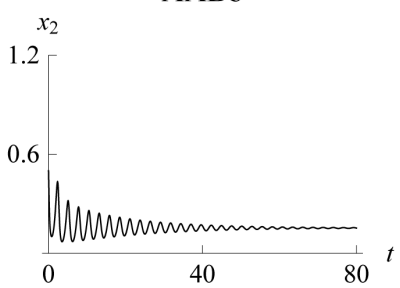

$\mathrm{AaBb}$

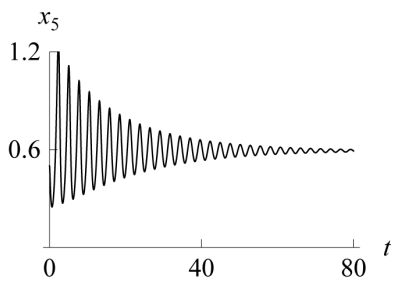

$\mathrm{aaBb}$

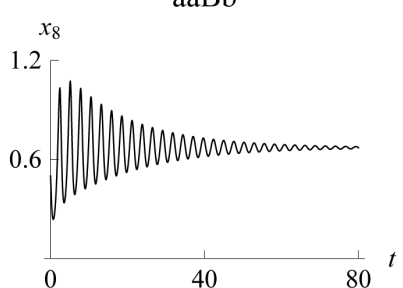

AAbb

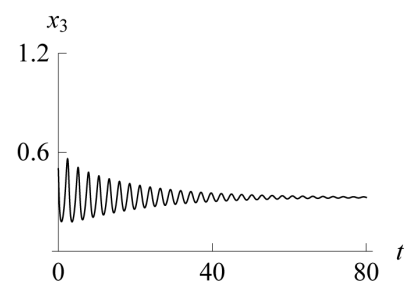

Aabb

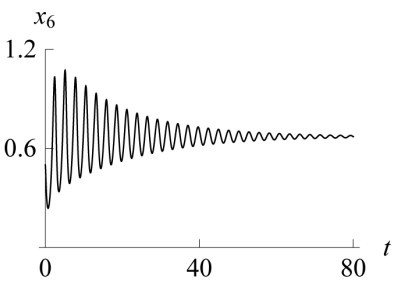

aabb

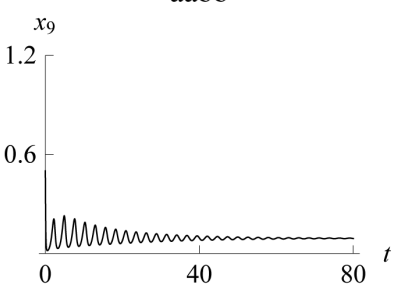

Figure 12. Stabilization with $k_{1}=3, k_{2}=1.2, k_{4}=1.2, k_{5}=1.2, l_{9}=3, m_{3}=0.8, m_{6}=0.8, f_{7}=0.8, f_{8}=0.8$.

and $a a B b$ (expected phenotype is $A b$ and $a B$ respectively) have the highest rates and more chances for survival.

\subsection{Example 2. (b) $k_{1}$ and $l_{9}$ Are the Greatest While All Other Parameter Values Are 1}

This case is interesting by the "slowness" in the rate at which the system stabilizes when compared to the previous two examples. From Figure 13 we see that both populations need around 170 steps to stabilize at one point.

This example numerically indicates that all genotypes except $A A B B$ and $a a b b$ find stabilization at the hight value population rate point. Both types $A A B B$ with dominant alleles and the weakest genotype $a a b b$ with recessive alleles have high catchability value $k_{1}=l_{9}=3$ and stabilize at the point close to zero (that is, they are close to extinction) (Figure 14). 

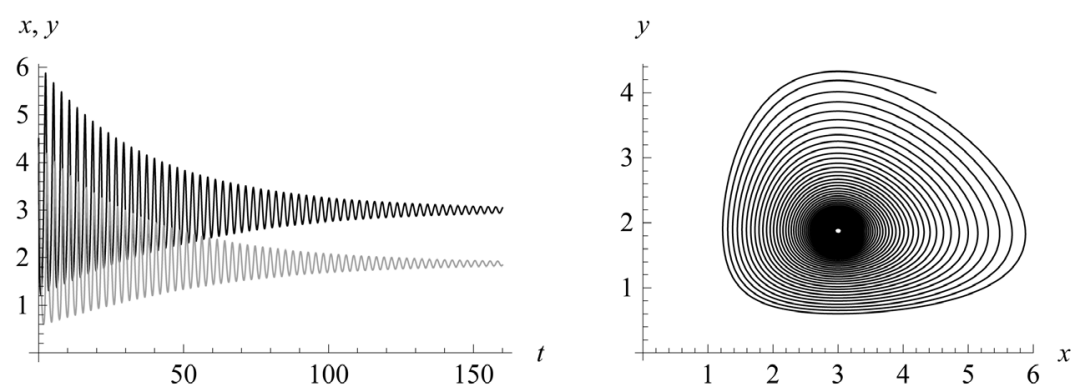

Figure 13. Stabilization with $k_{1}=3, k_{2}=1, k_{4}=1, k_{5}=1, l_{9}=3, m_{3}=1, m_{6}=1, f_{7}=1, f_{8}=1$.

$\mathrm{AABB}$
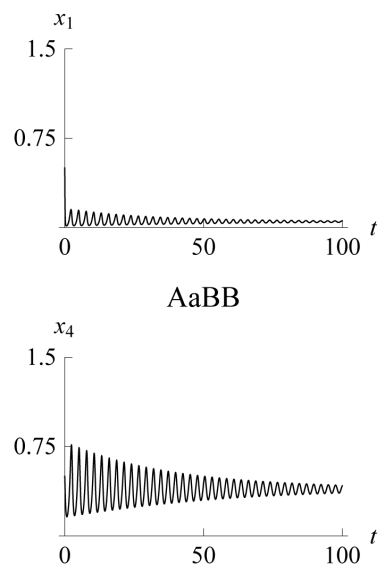

aaBB

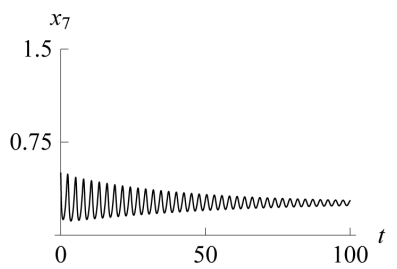

$\mathrm{AABb}$
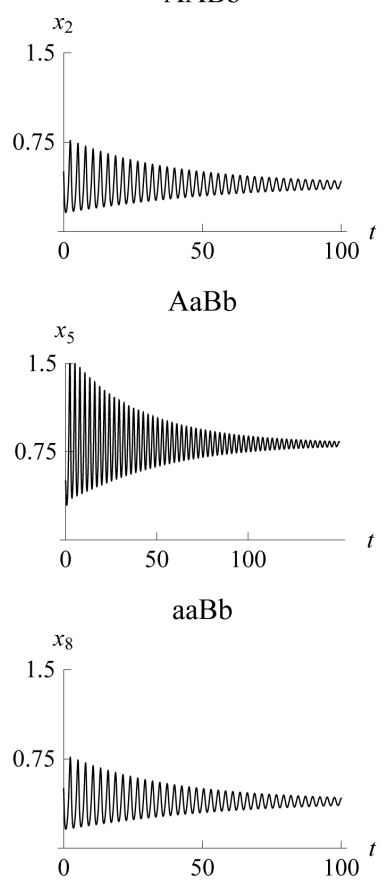

$\mathrm{AAbb}$
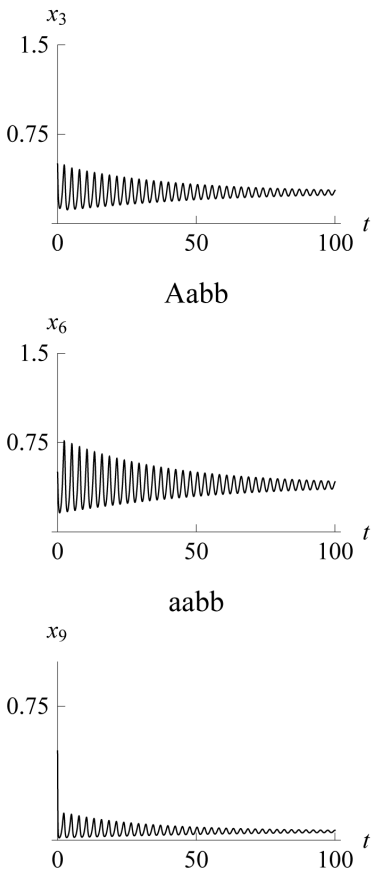

Figure 14. Stabilization with $k_{1}=3, k_{2}=1, k_{4}=1, k_{5}=1, l_{9}=3, m_{3}=1, m_{6}=1, f_{7}=1, f_{8}=1$.

\subsection{Example 3. $k_{1}$ Is the Smallest: The Catchability of the Organism with Genotype $A A B B$ Is the Lowest}

Setting $k_{1}$ smaller than the other $k_{i}$ values models epistasis by giving the genotype $A A B B$ (expected phenotype $A B$ ) a lower prey-induced death rate than the other organisms with phenotype $A B$. Figure 15 illustrates that the population/density rate of the prey changes from approximately $x=1.5$ to $x=5.3$ while the predator population/density rate changes from approximately $y=1.2$ to $y=5.6$.

Setting $k_{5}$ smaller than the other $k_{i}$ values models epistasis by giving the genotype $A a B b$ (expected phenotype $A B$ ) a lower prey-induced death rate than the other organisms with phenotype $A B$. The example illustrates an interesting situation. The small catchability rate of the species with genotype $A A B B$ with dominant alleles $A$ and $B$ and the same catchability rates for the other species with genotypes $A A B B$ survive the competition between the other genotypes and forces them to extinction. A critical point around $t=10$ can be observed. In this case, the $A$ and $B$ alleles go to fixation (Figure 16 and Figure 17).

\subsection{Example 4. $k_{5}$ Is the Smallest; $I_{9}$ Is the Greatest}

Both predator and prey populations stabilize at almost the same rate (around 2.6 for the predator and 3 for the prey with the parameter values we use) as shown in Figure 18. 

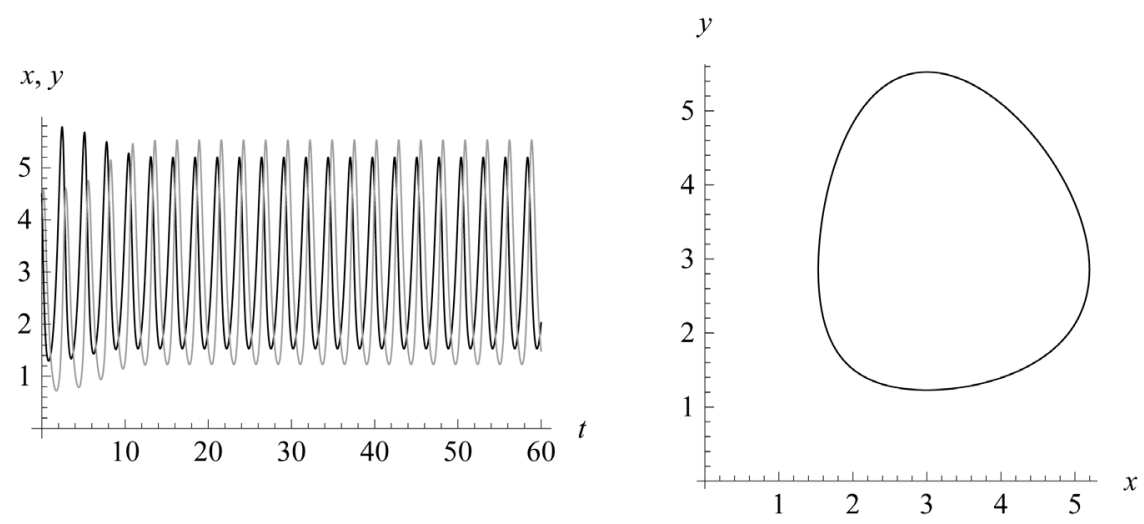

Figure 15. Limit cycle with $k_{1}=0.7, k_{2}=1, k_{4}=1, k_{5}=1, l_{9}=1, m_{3}=1, m_{6}=1, f_{7}=1, f_{8}=1$. The $A$ and $B$ alleles go to fixation so the genotype $A A B B$ is the only one to survive.

$\mathrm{AABB}$

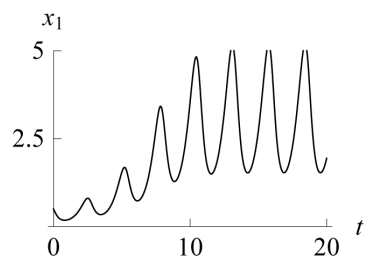

AaBB
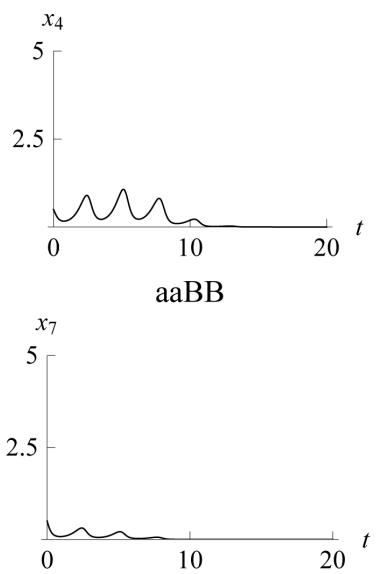

$\mathrm{AABb}$

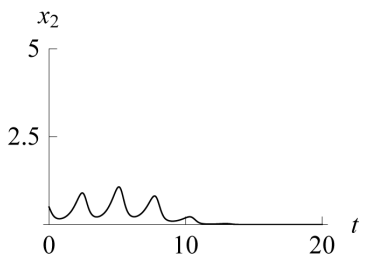

$\mathrm{AaBb}$

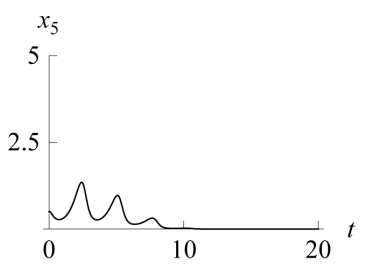

$\mathrm{aaBb}$

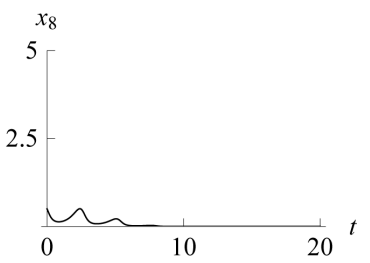

$\mathrm{AAbb}$

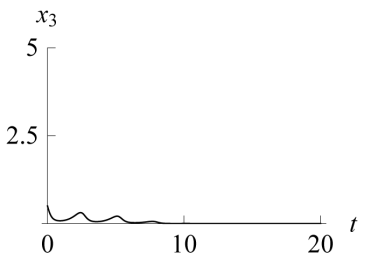

Aabb
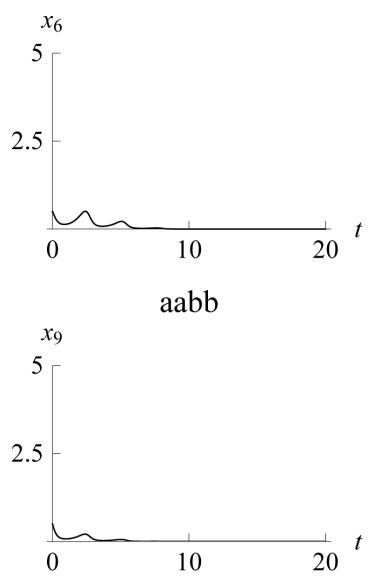

Figure 16. Limit cycle with $k_{1}=0.7, k_{2}=1, k_{4}=1, k_{5}=1, l_{9}=1, m_{3}=1, m_{6}=1, f_{7}=1, f_{8}=1$. The $A$ and $B$ alleles go to fixation to the genotype $A A B B$ is the only one to survive.

When the growth advantage is given to the $x_{5}$ organism (with genotype $A a B b$ ) and because random mating of the organism with genotype $A a B b$ produces all of other genotypes, we observe the stabilization shown in Figure 19. Since $x_{5^{\prime}}=2 a x(p s+r q)-k_{5} x_{5} y$, all other genotypes stabilize as well.

\subsection{Example 5. (a) $l_{9}$ Is the Largest; All Other Parameter Values Are Equal}

With these parameter values, the example, which is graphically illustrated in Figure 19, illustrates how the $k$ phenotype group $\left(x_{1}, x_{2}, x_{4}\right.$, and $\left.x_{5}\right)$ can be the strongest (or survive with the highest population/density) with respect to the population sizes/densities of the other genotypes. In this case, the $A$ and $B$ alleles go to fixation.

Making the weakest species with genotype $a a b b$ (because the genes $a$ and $b$ are recessive) more catchable by choosing large catchability parameter values forces the genotype to extinction. We have also observed 

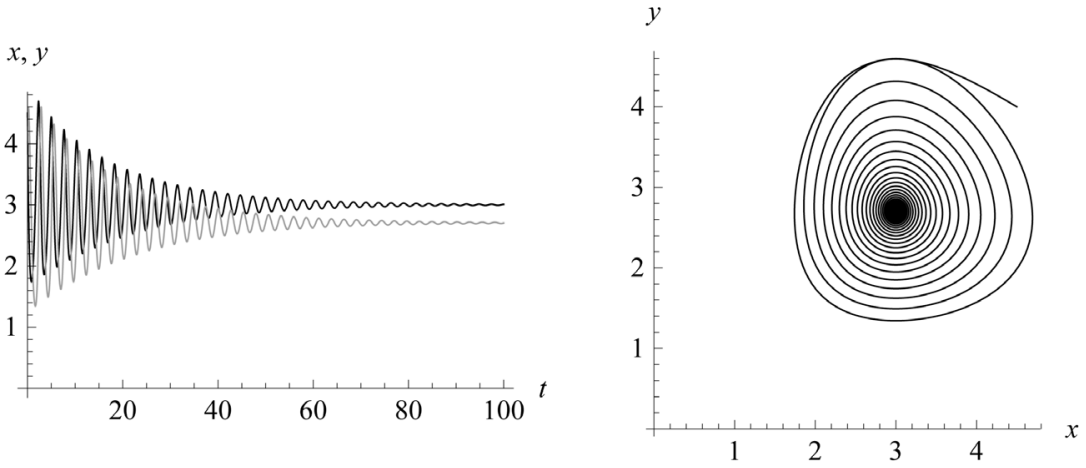

Figure 17. Stabilization with $k_{1}=1, k_{2}=1, k_{4}=1, k_{5}=0.5, l_{9}=3, m_{3}=0.75, m_{6}=0.75$, $f_{7}=0.75, f_{8}=0.75$.

\section{AABB}

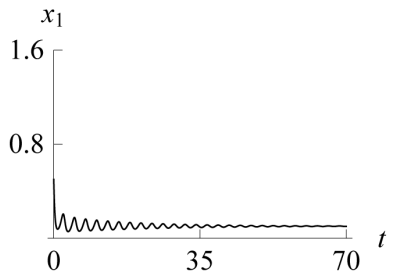

$\mathrm{AaBB}$

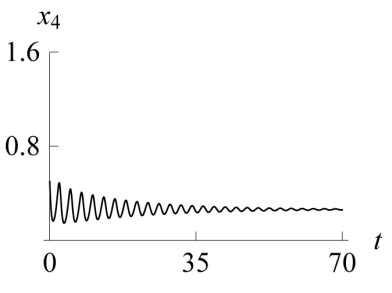

$\mathrm{aaBB}$

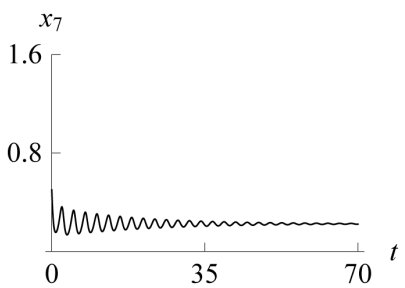

$\mathrm{AABb}$

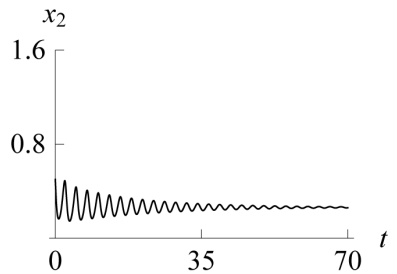

$\mathrm{AaBb}$
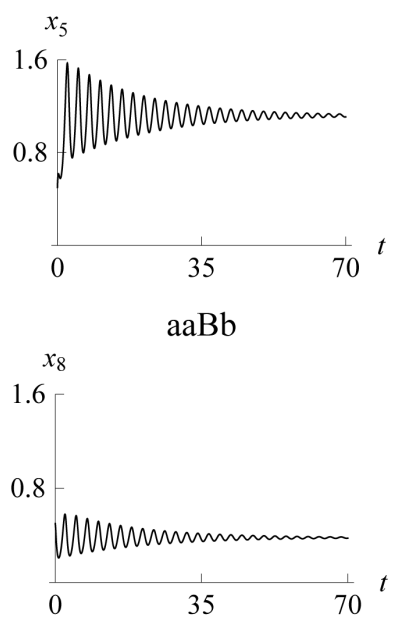

AAbb

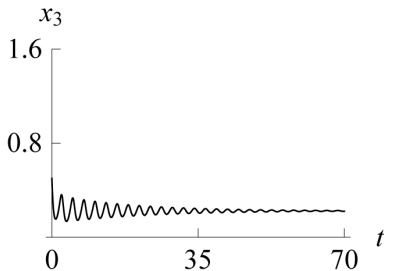

Aabb
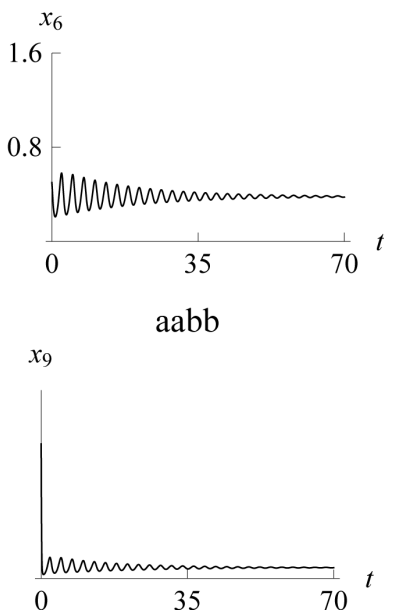

Figure 18. Stabilization with $k_{1}=1, k_{2}=1, k_{4}=1, k_{5}=0.5, l_{9}=3, m_{3}=0.75, m_{6}=0.75, f_{7}=0.75, f_{8}=0.75$.
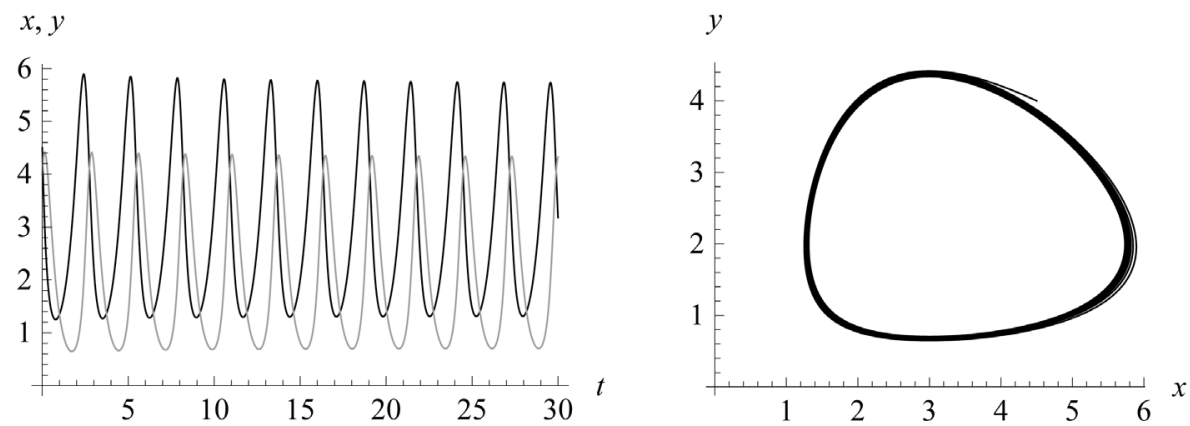

Figure 19. Limit ycle with $k_{1}=1, k_{2}=1, k_{4}=1, k_{5}=1, l_{9}=3, m_{3}=1, m_{6}=1, f_{7}=1, f_{8}=1$. 
that a limit cycle sometimes occurs. Population sizes/densities of the species with genotypes $A A B b$ and $A a B B$ decrease extremely slowly with the selected parameter values as shown in Figure 20.

\subsection{Example 5. (b) Parameter Value $I_{9}$ Is the Greatest and We Choose the Parameter Values $m_{6}$ and $f_{8}$ to Be Smaller}

As in the previous example (refer to Figure 17 and Figure 18), high population size/density of the organism with genotype $A a B b$ forces the system to stabilize. In this case, the organisms with genotypes $A a B b, A a b b$ and $a a B b$ have the highest values because their catchability parameters are the lowest with respect to the other population sizes/densities of the species with the other genotypes. The lowest population sizes/densities, as we have seen in the simulation, are the organisms with genotypes $A A B B$ and aabb (both around 0.1) (Figure 21 and Figure 22).
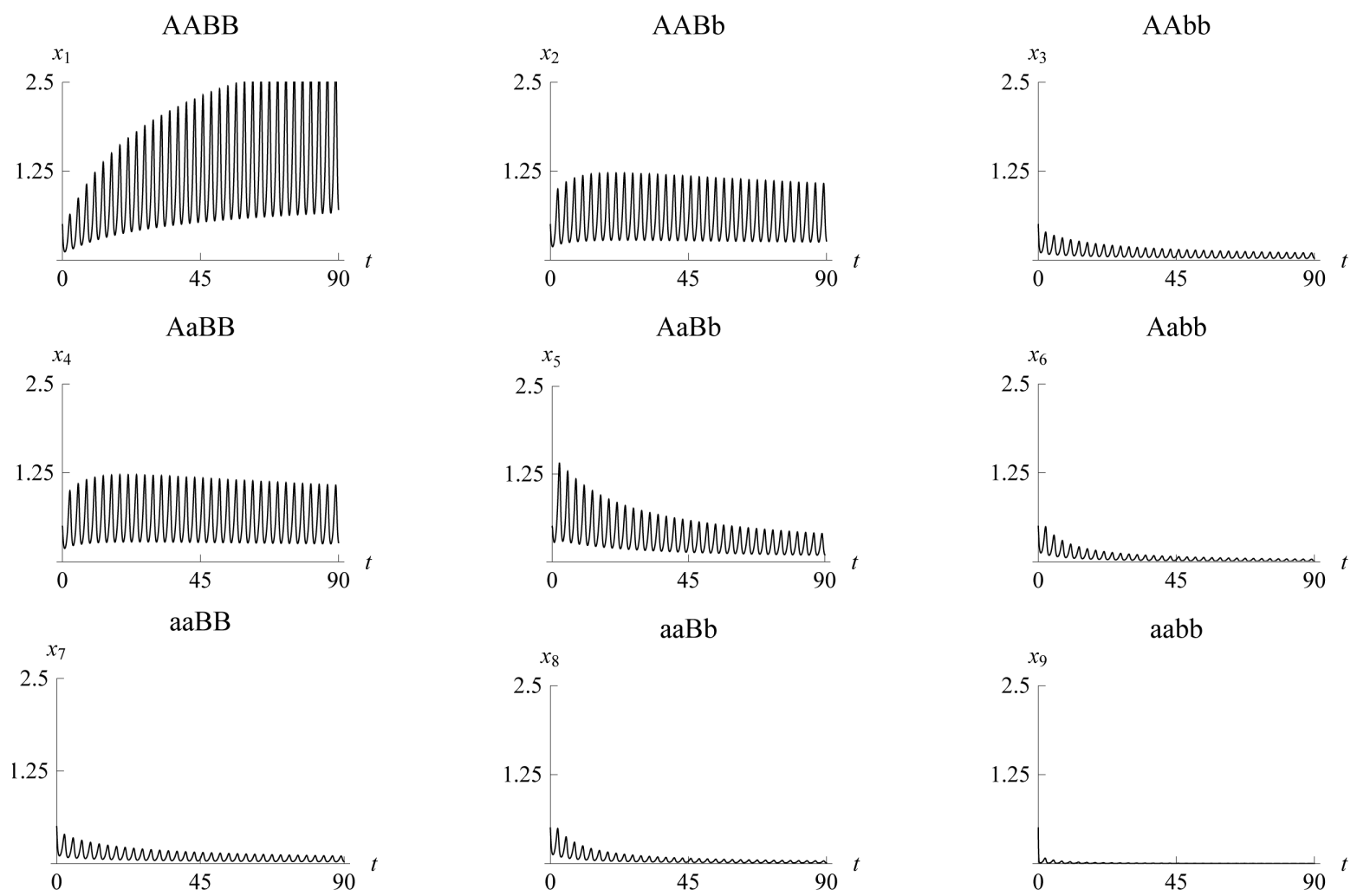

Figure 20. Limit cycle with $k_{1}=1, k_{2}=1, k_{4}=1, k_{5}=1, l_{9}=3, m_{3}=1, m_{6}=1, f_{7}=1, f_{8}=1$.
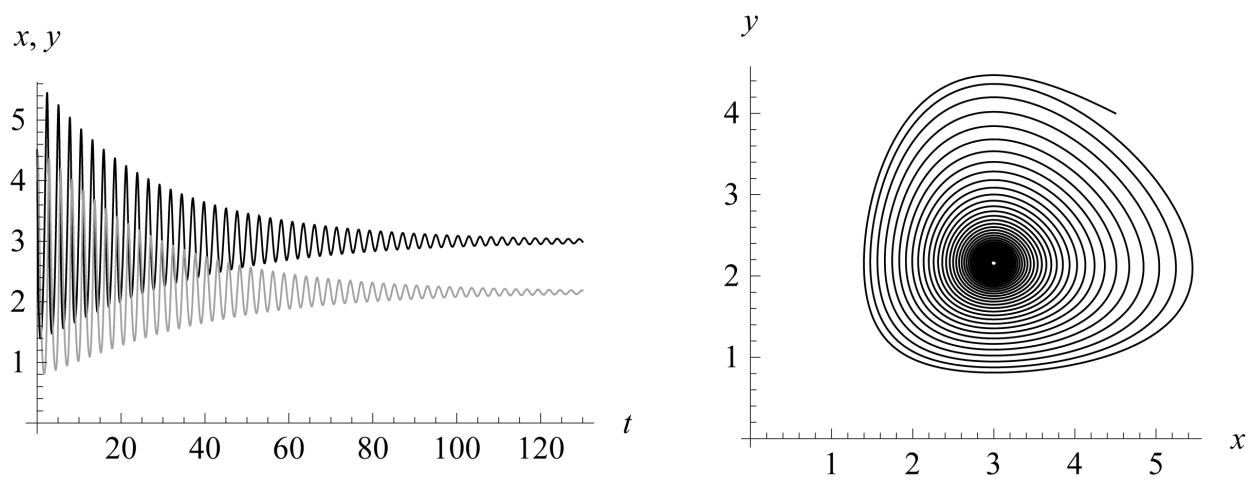

Figure 21. Stabilization with $k_{1}=1, k_{2}=1, k_{4}=1, k_{5}=1, l_{9}=3, m_{3}=1, m_{6}=0.7, f_{7}=1, f_{8}=0.7$. 
$\mathrm{AABB}$

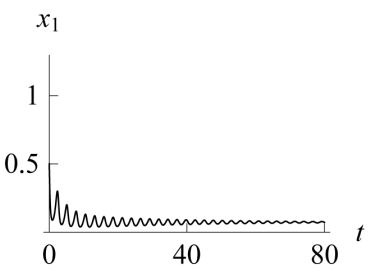

AaBB
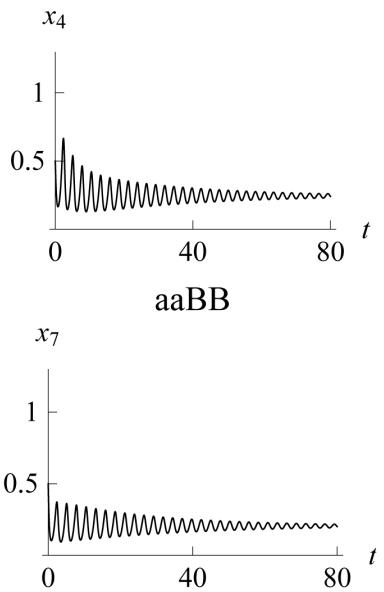

$\mathrm{AABb}$

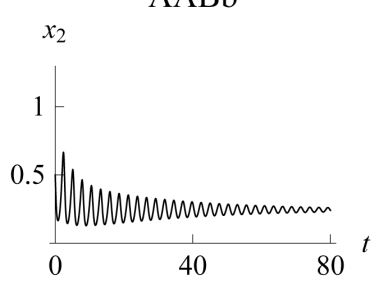

$\mathrm{AaBb}$
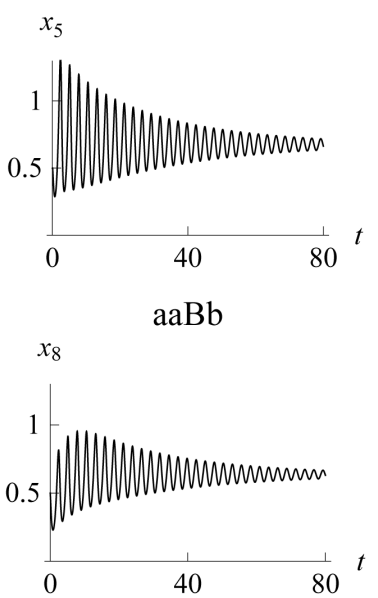

$\mathrm{AAbb}$

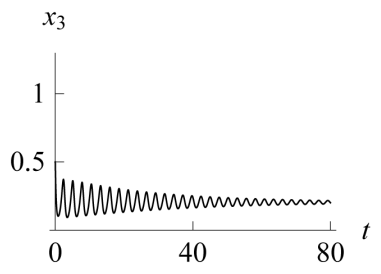

Aabb
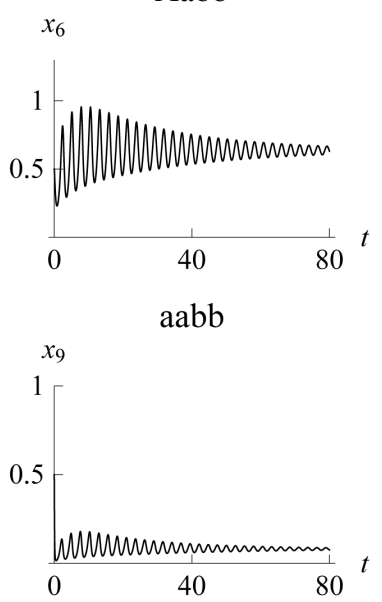

Figure 22. Stabilization with $k_{1}=1, k_{2}=1, k_{4}=1, k_{5}=1, l_{9}=3, m_{3}=1, m_{6}=0.7, f_{7}=1, f_{8}=0.7$.

\section{Conclusions}

In this paper we have discussed different cases of epistasis of the prey in a two-locus, two-allele problem in a basic predator-prey relationship. After discussing the most famous example of epistasis in humans, The Bombay Phenotype, we constructed the main model for a two-locus, two-allele problem with nine genotypes. Then we used different values for "catchability" parameters to examine both population sizes as well as genotypic and phenotypic population/densities.

In our simulations and examples, we saw that in different situations limit cycles or stabilization can occur. The simulations showed that the model was highly sensitive to the different parameters. Some cases illustrated total extinction of the weakest types of the prey, while in other examples all population types survived and a limit cycle occurred. Some interesting examples were observed where the weakest or the strongest types also became extinct. Since random mating of the organism of type $A a B b$ produces all of the other genotypes (and phenotypes), this type had the highest population rate most of the time.

Future studies might include using different predator-prey models such as epistasis of the predator in a twolocus two-allele problem, epistasis of both predator and prey in the same model, or epistasis in the dangerous predator case. In future studies, we will be able to see how the numerical results obtained here might change or how the situation might evolve differently if epistasis occurs in both the predator and the prey. Other interesting studies would be to incorporate epistasis into competition, cooperation problems, or host-parasite problems.

\section{Computational Remarks}

Mathematica 9.0 [9], was used to create the graphics and perform the computations presented in this paper. Copies of the Mathematica notebooks used are available from the authors by sending a request for them to Jim Braselton at jbraselton@georgiasouthern.edu.

\section{References}

[1] Karlin, S. (1972) Some Mathematical Models of Population Genetics. American Mathematical Monthly, 79, $699-739$. http://dx.doi.org/10.2307/2316262 
[2] Dean, L. (2005) Blood Groups and Red Cell Antigens. National Center for Biotechnology Information.

[3] Lotka, A.J. (1956) Elements of Mathematical Biology. Dover, New York

[4] Abell, M. and Braselton, J. (2010) Introductory Differential Equations with Boundary Value Problems. 3rd Edition, Academic Press, Boston.

[5] Beltrami, E. (2013) Mathematical Models for Society and Biology. 2nd Edition, Academic Press, Boston.

[6] Murray, J. (2007) Mathematical Biology: I. An Introduction. 3rd Edition, Springer, New York.

[7] Braselton, J., Abell, M. and Braselton, L. (2005) Selective Mating in a Continuous Model of Epistasis. Applied Mathematics and Computation, 171, 225-241. http://dx.doi.org/10.1016/j.amc.2005.01.059

[8] Szathmáry, E. (1993) A Note on the Reduction of the Dynamics of Multilocus Diploid Genetic Systems with Multiplicative Fitness. Journal of Theoretical Biology, 164, 351-358. http://dx.doi.org/10.1006/jtbi.1993.1159

[9] Wolfram Research, Inc. (2013) Dominance, Population Size, and Delayed Inheritance. Evolution, 67, 2011-2023. 


\section{Appendix: Ratios of Offspring for $x_{i}-x_{j}$ Mating Combinations}

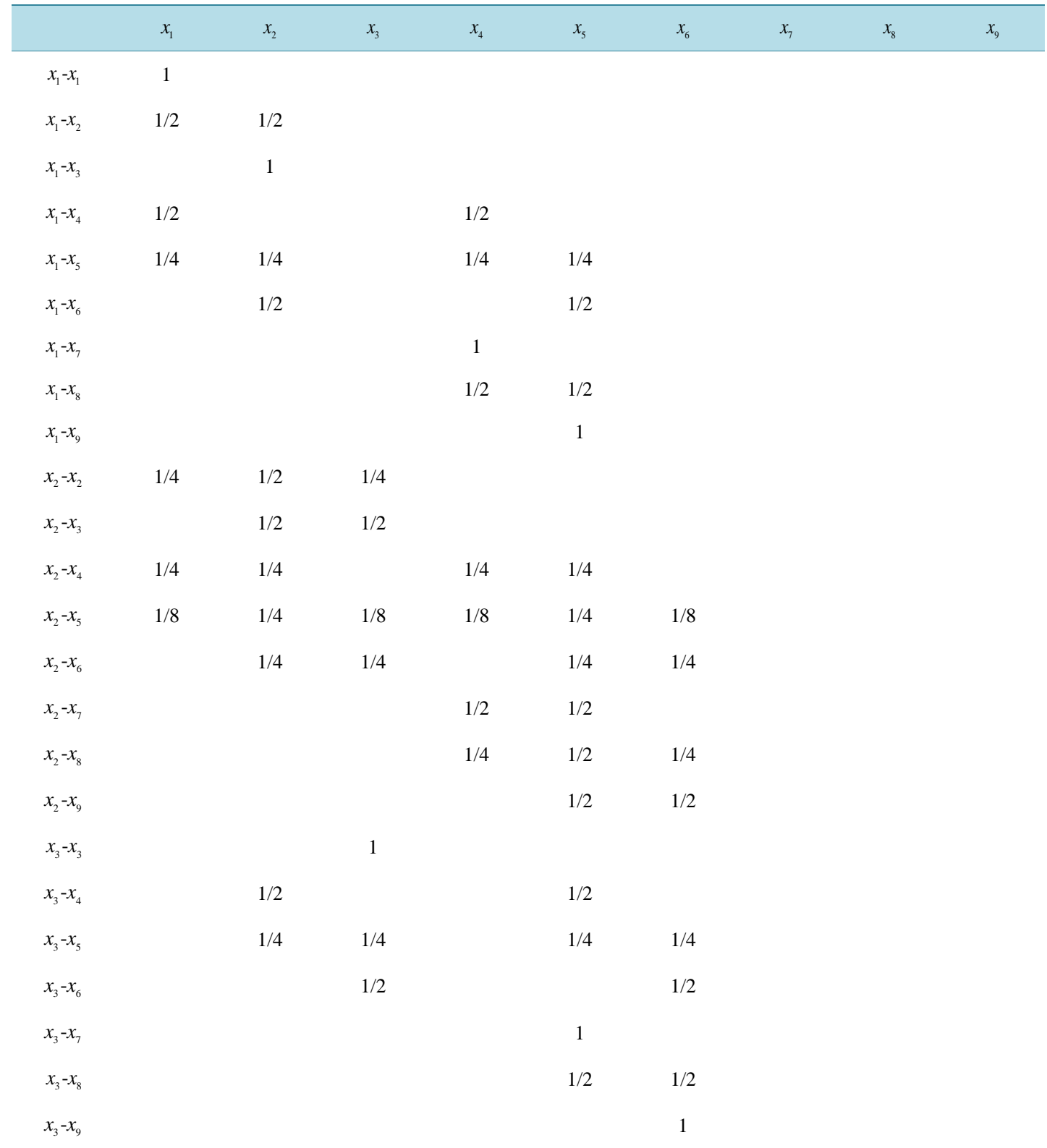

Table A1. Ratios of offspring for $x_{i}-x_{j}$ mating combinations.

\begin{tabular}{|c|c|c|c|c|c|c|c|c|c|}
\hline$X_{4}-X_{4}$ & $1 / 4$ & & & $1 / 2$ & & & $1 / 4$ & & \\
\hline$X_{4}-X_{5}$ & $1 / 8$ & $1 / 8$ & & $1 / 4$ & $1 / 4$ & & $1 / 8$ & $1 / 8$ & \\
\hline$x_{4}-x_{6}$ & & $1 / 4$ & & & $1 / 2$ & & & $1 / 4$ & \\
\hline$x_{4}-X_{7}$ & & & & $1 / 2$ & & & $1 / 2$ & & \\
\hline$X_{4}-X_{8}$ & & & & $1 / 4$ & $1 / 4$ & & $1 / 4$ & $1 / 4$ & \\
\hline$X_{4}-X_{9}$ & & & & & $1 / 2$ & & & $1 / 2$ & \\
\hline$x_{5}-x_{5}$ & $1 / 16$ & $1 / 8$ & $1 / 16$ & $1 / 8$ & $1 / 4$ & $1 / 8$ & $1 / 16$ & $1 / 8$ & $1 / 16$ \\
\hline
\end{tabular}


I. Inozemtseva, J. Braselton

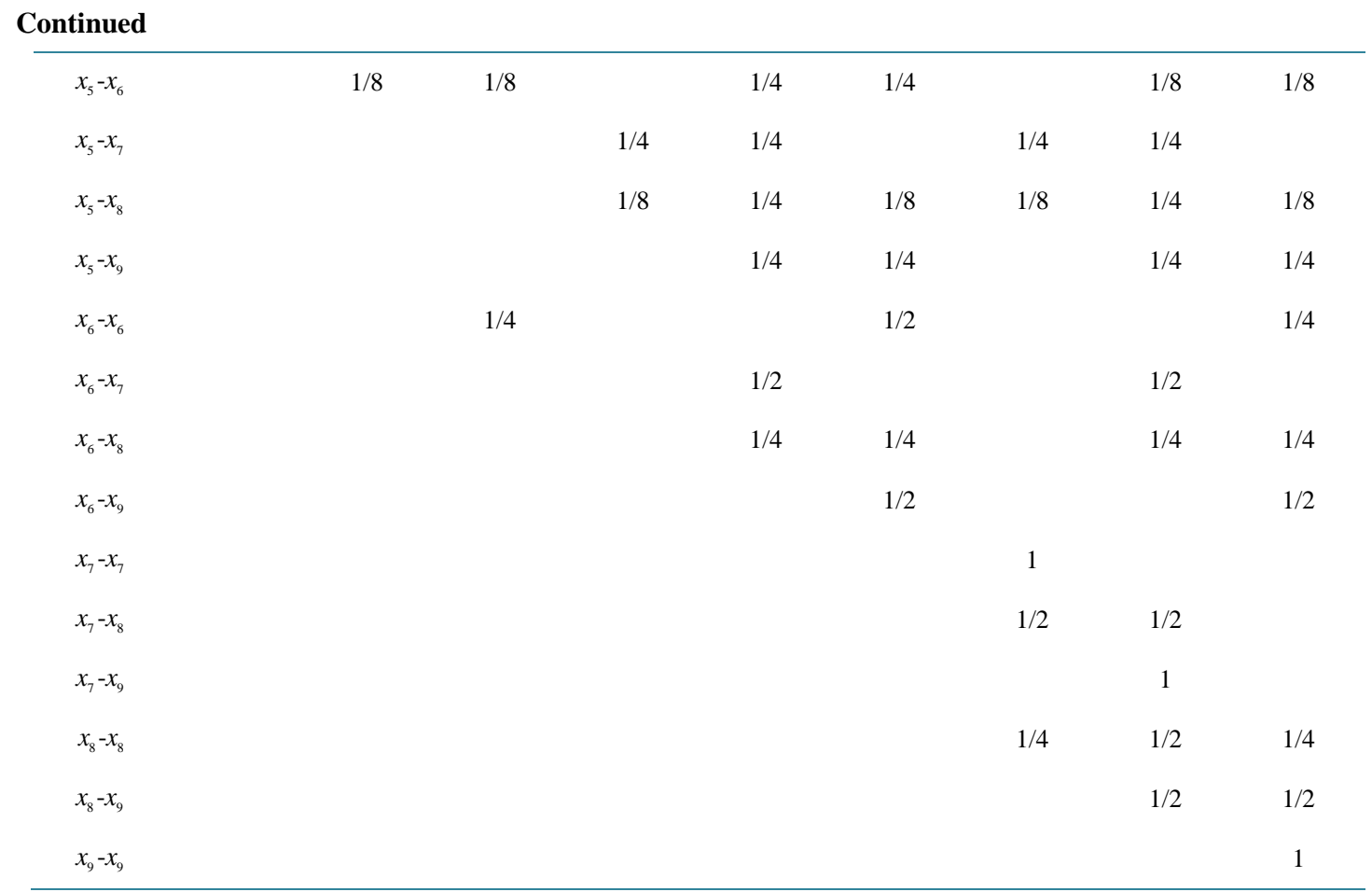


Scientific Research Publishing (SCIRP) is one of the largest Open Access journal publishers. It is currently publishing more than 200 open access, online, peer-reviewed journals covering a wide range of academic disciplines. SCIRP serves the worldwide academic communities and contributes to the progress and application of science with its publication.

Other selected journals from SCIRP are listed as below. Submit your manuscript to us via either submit@scirp.org or Online Submission Portal.
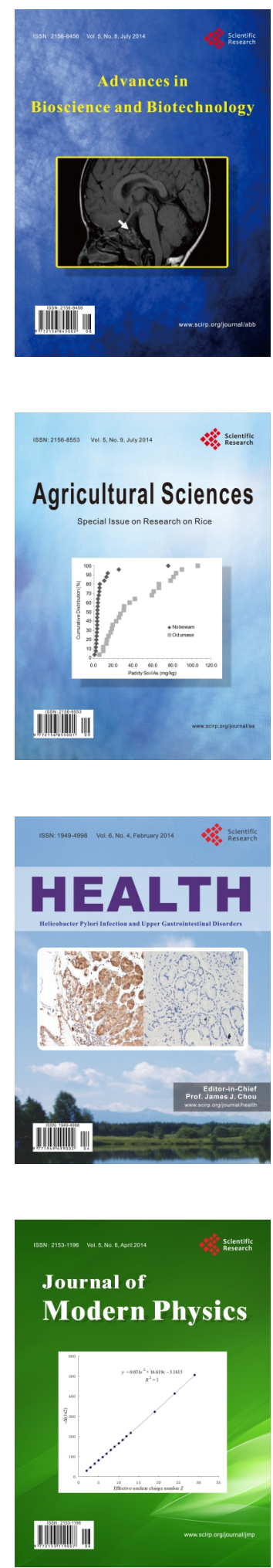
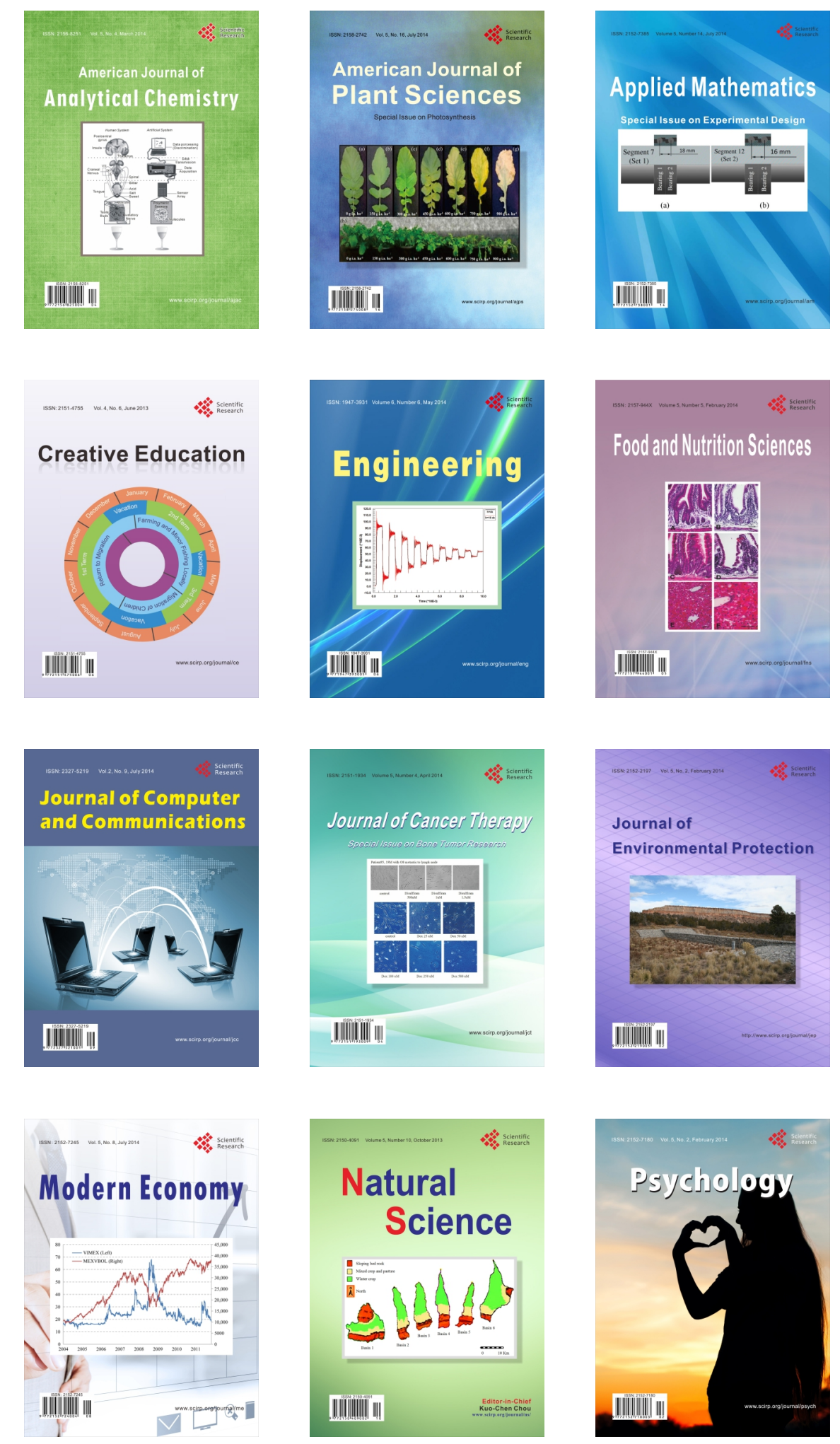